\title{
Fermented soyabean products as hypoallergenic food
}

\author{
J. Frias $^{1}$, Y. S. Song ${ }^{2}$, C. Martínez-Villaluenga ${ }^{1}$, E. Gonzalez de Mejia ${ }^{2}$ and C. Vidal-Valverde ${ }^{1}$ \\ ${ }^{1}$ Instituto de Fermentaciones Industriales (CSIC), Juan de la Cierva 3, 28006 Madrid, Spain and ${ }^{2}$ Department of Food \\ Science and Human Nutrition, University of Illinois Urbana-Champaign, 228 ERML, MC-051, 1201 W Gregory Drive,
}

Urbana, IL 61801, USA

Food allergy has become a public health problem that continues to challenge both the public and the food industry, and soyabean is causative of food allergy. The allergenicity of food proteins has been shown to be modified by food-processing technologies such as fermentation. The objective of the present research was to ferment soyabeans as flour (liquid fermentation) or as cracked seeds (solid fermentation) using different micro-organisms in order to study the effect on immunoreactivity after fermentation. Immunochemical methods have been developed and validated for the detection and quantification of the major human allergenic soyabean proteins, and a comparison with fermented soyabean proteins has been carried out. ELISA and Western blot were used to quantify IgE antibody response and HPLC to identify and quantify total amino acids. The extractable protein concentration decreased after fermentation (from $31 \%$ for cracked seeds fermented with Bacillus subtilis to $74 \%$ with Aspergillus oryzae). Lactobacillus plantarum- and naturally-fermented soyabean flour showed a higher reduction in IgE immunoreactivity ( $\leq 92 \%$ and $97 \%$ ) in comparison with the corresponding fermented products of cracked seeds ( $92 \%$ and $88 \%$ reduction respectively). Among the solid fermented products, the lowest performance was given by mould strains, Rhizopus oryzae and A. oryzae, which showed a reduction in allergenic values of $66 \%$ and $71 \%$ respectively. Although B. subtilis showed the highest soluble protein concentration $(221.5 \mathrm{mg}$ protein/g product), this fermented soyabean product yielded a $75 \%$ and $86 \%$ reduction in immunoreactivity against human plasma and human pooled plasma samples respectively.

The raw soyabean flour presented the highest complexity on protein profile and immunoreactivity. Human plasma samples presented an intense immunoreactivity towards specific immunodominant proteins. Fermentation with B. subtilis or L. plantarum and natural fermentation yielded peptides of smaller size and less-intense immunoreactivity. Moreover, soyabeans subjected to fermentation processes showed rises for most of the non-essential and essential amino acids $(P \leq 0.05)$. In conclusion, fermentation applied to soyabean induces a noticeable variation in protein profile and allergenicity and there is potential for developing nutritious hypoallergenic soya products.

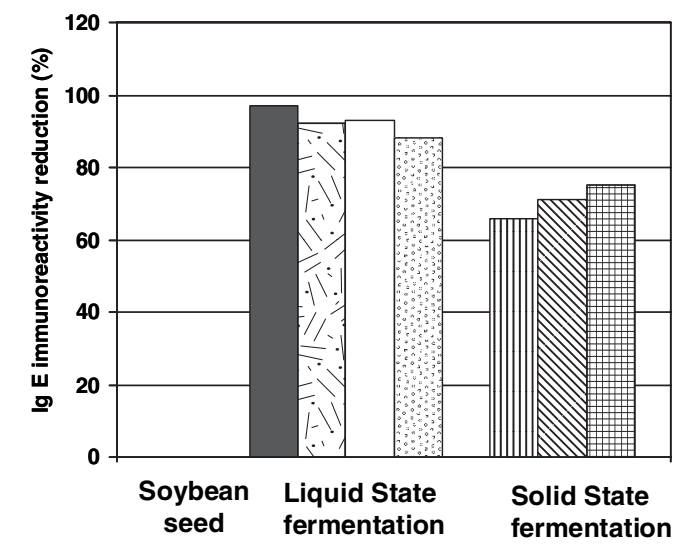

LIQUID STATE

- L. plantarum (soy flour)

$\square$ L. plantarum (cracked soy)

$\square$ Natural (soy flour)

Natural (cracked soy)

SOLID STATE

四 A. oryzae (cracked soy)

$\mathbb{Q}$ R. oryzae (cracked soy)

B. subtilis (cracked soy)

Figure 1. Effect of fermentation of soybean seeds and soya fermented products on immune response by ELISA.

This work was partly funded by AGL2004-0886ALI and by USDA-Future Foods Initiative. 